\title{
Interpretation of Local Flux Measurements in Subcritical Systems and Reactivity Determination
}

\author{
S. Dulla and P. Ravetto \\ Dipartimento Energia, Politecnico Di Torino, Corso Duca degli Abruzzi, 24-10129 Torino, Italy \\ Correspondence should be addressed to S. Dulla, sandra.dulla@polito.it
}

Received 9 January 2012; Accepted 28 February 2012

Academic Editor: Alberto Talamo

Copyright ( $) 2012$ S. Dulla and P. Ravetto. This is an open access article distributed under the Creative Commons Attribution License, which permits unrestricted use, distribution, and reproduction in any medium, provided the original work is properly cited.

\begin{abstract}
The determination of the subcriticality level constitutes an important issue in the assessment of the accelerator-driven system technology. For this purpose, the interpretation of flux measurements requires a lumped-parameter model employed in an inverse fashion. This papers addresses the drawbacks of point kinetics in performing such a task. In particular, the problem of the generation of integral parameters is considered, in connection with the use of a shape function and of a projection weight tailored to the neutron flux detector. Furthermore, the question of the generation of the effective source is analysed, and some proposals to modify the time dependence of such a function to account for the time delay at the flux detector are presented and discussed.
\end{abstract}

\section{Introduction}

The assessment of the feasibility of subcritical acceleratordriven systems requires the performance of experiments to verify the kinetic response features and the determination of the integral parameters that characterize the physical multiplying structure. Typical experiments involve the measurements of flux signals during source or reactivity-induced transients, either in pulsed or oscillated modes. Several experimental campaigns have been conducted in the past years $[1,2]$, and new more ambitious and advanced experiments are programmed in the near future.

An issue that is particularly important is the monitoring of the subcriticality level of the reactor. It is, therefore, required to establish efficient procedures to interpret flux measurements retrieved from detectors localized in various positions of the structure. This constitutes a basic and challenging inverse problem in reactor physics since the early times of nuclear energy and various approaches have been developed [3-5]. The interpretation of local measurements may be carried out only through suitable physicomathematical models. The inverse procedure can be easily implemented if a lumped-parameter model, such as classic point kinetics, is adopted. However, usually only localized values of the neutron flux are available from the detectors. Hence, the flux interpretation scheme needs unavoidably to account for spectral and spatial effects. It has been shown [6] by numerical simulations that the point model with integral kinetic parameters may be inadequate when local flux signals are used in a point framework to reconstruct the global system reactivity in an inverse fashion, while it may perform rather well for the analysis of the full power evolution, which is, however, seldom available from the direct experimental information. This aspect is also confirmed by real experimental analyses [7].

From the theoretical point of view, to construct integral kinetic parameters a projection involving the neutronic shape over a weighting function is always required. While for critical systems the mathematically consistent and physically meaningful choice for the weighting function is unique, as the reference steady-state system adjoint (neutron importance) [8], for source-driven systems the necessity of a definition of the adjoint source introduces a certain degree of freedom [9]. It has been shown [10] that the effect of the choice of the weighting function may be quite significant. The freedom of the choice may be used to suit the objectives 
of the analysis. To be more specific, the adjoint source can be assumed as a cross section characterizing the physical properties of the local detector where the flux measurement is taken. The present paper shall discuss the effectiveness of the weighting to simulate in a point-like manner the evolution of local flux signals, therefore envisaging the possibility of devising tailored point models to interpret experimental measurements and establish the limits of the technique.

Another aspect that plays an important role in the interpretation procedure for source transients is the suitable timeshape of the neutron source that is assumed in the lumpedparameter model [11]. It is obvious that a time delay needs to be introduced when analyzing the signal from detectors away from the source region. This aspect is also discussed in the following.

\section{The Factorization-Projection Procedure for the Neutronic Equations}

A lumped-parameter model is derived from the most general balance equations involving both neutrons and delayed neutron precursors. By indicating with $n(\mathbf{x}, t)$ the neutron density at phase point $\mathbf{x}$ at time $t$, the balance equations can be written as:

$$
\begin{gathered}
\frac{\partial n}{\partial t}=\widehat{L} n+\hat{F}_{p} n+\lambda C+S \\
\frac{\partial C}{\partial t}=-\lambda C+\hat{F}_{d} n
\end{gathered}
$$

where only one family of delayed neutron precursors is considered, being the extension to any number of families just trivial. The symbols $\widehat{L}, \hat{F}_{p}$, and $\widehat{F}_{d}$ denote the leakage, prompt neutron production, and delayed neutron production operators, respectively. They may take appropriate forms consistent with the neutronic model adopted (e.g., discrete ordinate approach, spherical harmonics, diffusion) to treat the transport process. The quantity $S$ indicates the external neutron source. A factorization is now introduced according to the classical procedure [12]:

$$
n(\mathbf{x}, t)=A(t) \varphi(\mathbf{x}, t) .
$$

A projection step leads to the required model, introducing integral parameters that are constant in time if the shape function and the system properties are assumed to be constant. The operation involves the multiplication of each equation by a proper weighting function followed by integration over phase space. When treating initially critical systems, the importance function, solution of the adjoint homogeneous equation [13], is used. For subcritical systems, the weighting function can be chosen according to the solution of an adjoint source-driven problem [10]:

$$
\hat{L}_{0}^{\dagger} \phi_{S}^{\dagger}+\hat{F}_{0}^{\dagger} \phi_{S}^{\dagger}+S^{\dagger}=0
$$

where $\hat{L}_{0}^{\dagger}$ and $\hat{F}_{0}^{\dagger}$ are the adjoint destruction and production operators for the reference (initial) system, respectively.
The factorization followed by a projection operation upon a general weighting function $\psi$ leads to the following system of first-order differential equations in time:

$$
\begin{gathered}
\frac{d A}{d t}=\frac{\langle\psi \mid \hat{L} \varphi\rangle}{\langle\psi \mid \varphi\rangle} A+\frac{\left\langle\psi \mid \hat{F}_{p} \varphi\right\rangle}{\langle\psi \mid \varphi\rangle} A+\lambda \frac{\langle\psi \mid C\rangle}{\langle\psi \mid \varphi\rangle}+\frac{\langle\psi \mid S\rangle}{\langle\psi \mid \varphi\rangle}, \\
\frac{d}{d t} \frac{\langle\psi \mid C\rangle}{\langle\psi \mid \varphi\rangle}=\frac{\left\langle\psi \mid \hat{F}_{d} \varphi\right\rangle}{\langle\psi \mid \varphi\rangle} A-\lambda \frac{\langle\psi \mid C\rangle}{\langle\psi \mid \varphi\rangle} .
\end{gathered}
$$

The coefficients of such a system constitute the so-called kinetic parameters, which are perfectly defined once the shape and the adjoint functions are defined.

As already pointed out, there is some freedom in the choice of the adjoint source. The selection of the problemoriented adjoint source as the space- and energy-dependent cross section of the detector through which the local flux is measured may be physically advantageous for experimental interpretations. With this choice, the physical characteristics of the detector are accounted for. This model is denoted as local point kinetics (lpk) in the following. For other applications, in which the interest is more focused on the global behaviour of the system and thus the attention is concentrated on the fission process, the fission productivity $\nu \Sigma_{f}$ may be used, leading to a global point kinetic model $(g p k)$. Of course, there is always the possibility to choose the critical adjoint as the weighting function, leading to a critical point kinetic equation $(c p k)$.

Another important aspect in the generation of the lumped-parameter model concerns the choice of the shape function to be introduced in the factorization. Obviously, for a reactor departing from a criticality steady-state, the fundamental eigenfunction is naturally used, while for a deeply subcritical source-driven system at equilibrium the source-driven stationary distribution seems to be the most appropriate choice. However, a difficulty arises in the simulation of pulsed experiments, for which no neutrons are usually present in the initial system and no reference steady-state situation is approached during the experiment. Different kinetic models can thus be envisaged, by either using the critical shape, $\varphi_{c}$, or the source-driven shape, $\varphi_{s}$. All combinations of choices for the shape and for the adjoint are possible.

Summarizing the previous discussion, it is worth studying the performance of kinetic models obtained by the adoption of different shapes and weighting functions in the simulation of power transients and local flux signal evolutions, especially when the attention is focused on experimental analyses. Two shapes can be considered, the critical and the source-driven reference distributions. Furthermore, the weighting function can be chosen according to either the critical importance or the source-driven fission importance (the adjoint source is the system fission cross section), both retaining a global information, as well as a detector-tailored importance (the adjoint source is the detector cross section). In the following, some results are presented to highlight the features and the performance of the various possible models, which are quite numerous if one considers all the possible 


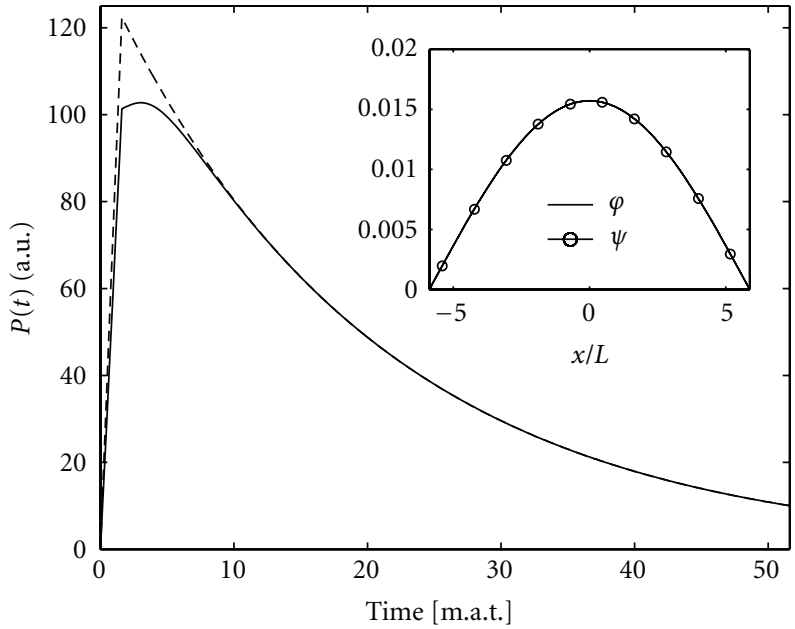

(a) $c p k$ and critical shape

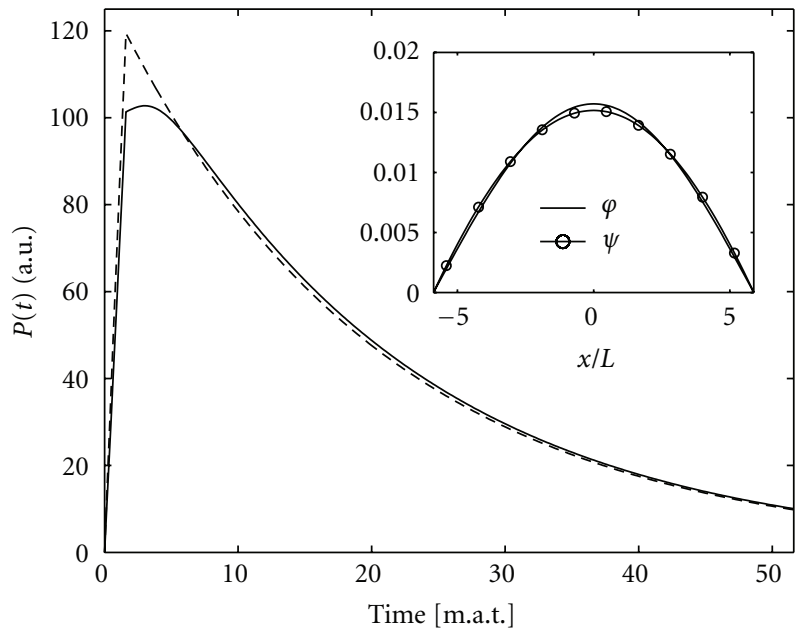

(c) gpk and critical shape

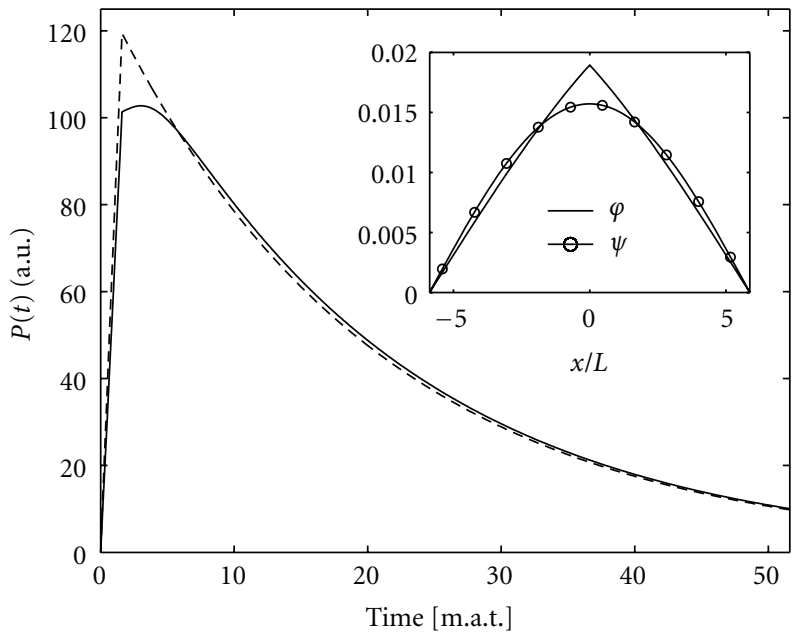

(b) $c p k$ and source-driven shape

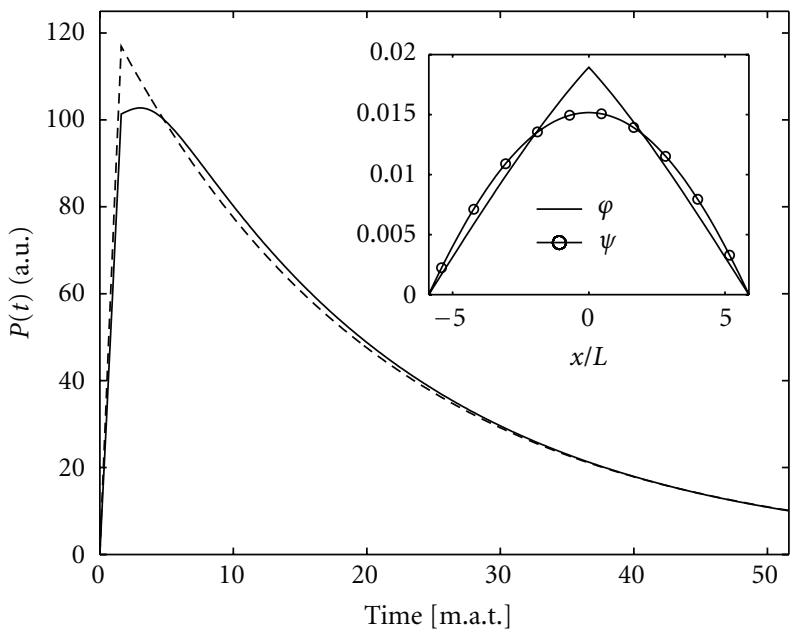

(d) $g p k$ and source-driven shape

FIGURE 1: Power evolution following a source pulse in a subcritical system $\left(k_{\text {eff }}=0.95\right)$, simulated with different approaches to point kinetics: $c p k$ and $g p k$. Solid line: reference; dashed line: point kinetics. The inset graphs represent the shape $\varphi$ and the weight $\psi$ adopted (m.a.t.: mean absorption time $\left.=1 /\left(\mathrm{v} \sigma_{a}\right)\right)$.

combinations. However, we restrict to presenting a limited number of cases, deemed to give some physical insight that may be useful to better understand the limits of a lumpedparameter model when used for experimental interpretations.

\section{Application of Local and Global Point Kinetics to the Interpretation of Pulsed Experiments}

In the following, comparisons between exact results for a pulsed source in a system described by one-group diffusion theory, assumed as the reference model, and the corresponding point kinetic model are presented. The system considered is characterized by the following physical parameters: neutron velocity $\mathrm{v}=10^{7} \mathrm{~cm} / \mathrm{s}$, diffusion coefficient $D=$ $1.5 \mathrm{~cm}$, diffusion length $L=10 \mathrm{~cm}$, and slab thickness
$H=117.5 \mathrm{~cm}$. The value of the infinite multiplication constant has been set in order to obtain the required $k_{\text {eff }}$, and two different subcriticality levels have been considered to highlight the effects of subcriticality. The source is localized and placed in the center of the system $(S(x)=\delta(x))$. In Figures $1-4$, the power evolution following a source pulse of duration $\tau=10 \mu$ s is presented, comparing the reference and point kinetic results, with different choices of the shape and weighting functions.

As can be seen in Figures 1 and 3, no relevant differences can be observed when adopting a critical or source-driven shape, and the adoption of a global ( $g p k)$ or critical (cpk) point kinetic model provides similar results in the reproduction of the power, with an average good quality, although the point models are not able to reproduce the behavior at short times, due to the presence of strong spatial effects in the propagation of the source pulse. On the other hand, 


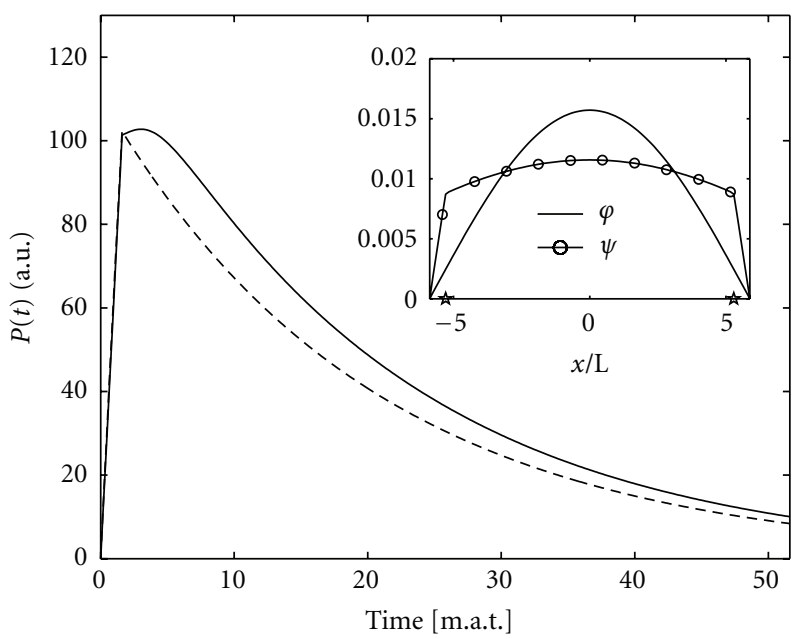

(a) lpk and critical shape $(\star$ : detector location)

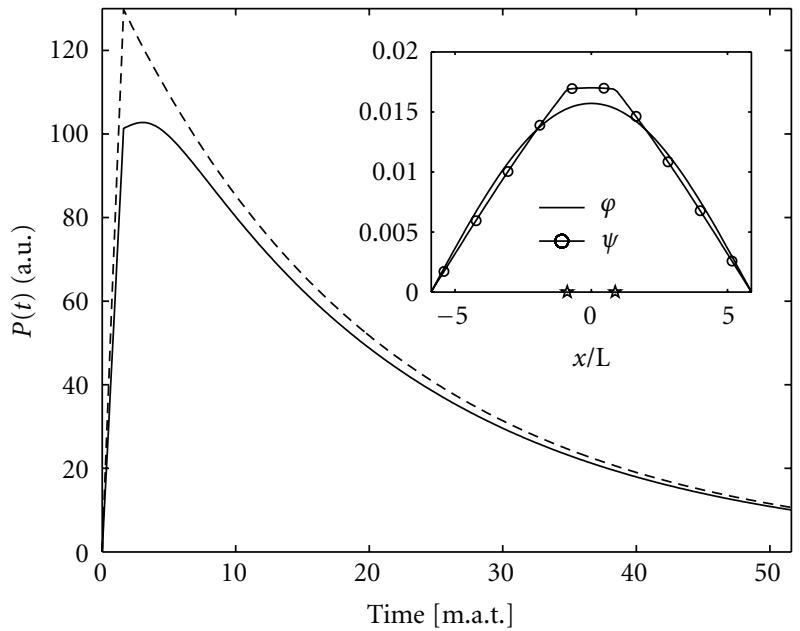

(c) lpk and critical shape ( $\star$ : detector location)

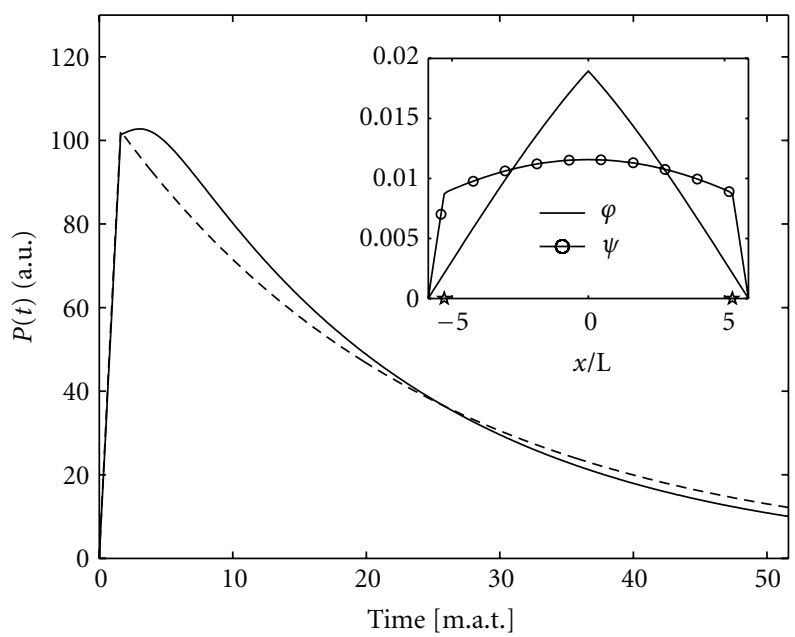

(b) $l p k$ and source-driven shape $(\star$ : detector location)

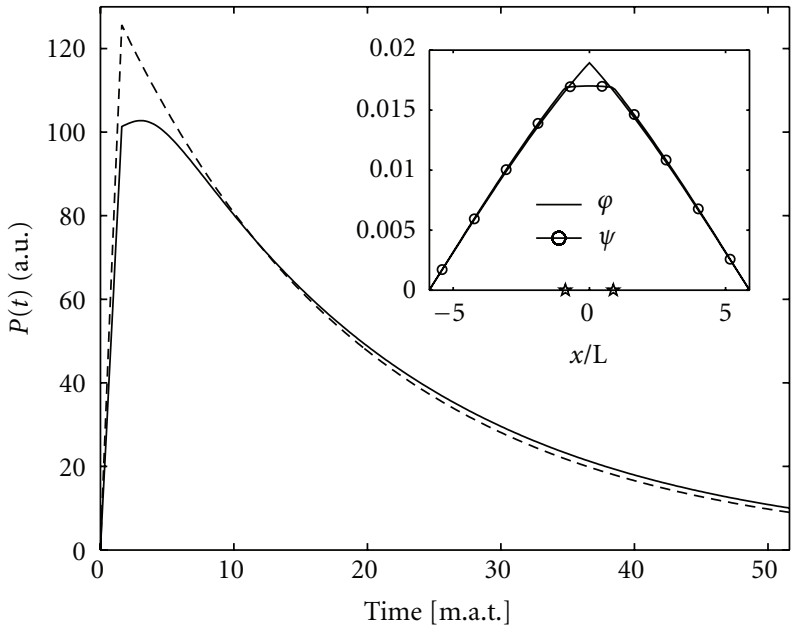

(d) lpk and source-driven shape ( $\star$ : detector location)

FIGURE 2: Power evolution following a source pulse in a subcritical system ( $k_{\text {eff }}=0.95$ ), simulated with a lpk approach, considering two different detector locations: first row, detector far from the source $\left(x_{0}=5.3 \mathrm{~L}\right)$; second row: detector close to the source $\left(x_{0}=0.9 \mathrm{~L}\right)$. Solid line: reference; dashed line: point kinetics. The inset graphs represent the shape $\varphi$ and the weight $\psi$ adopted.

the results obtained with the local point kinetics, presented in Figures 2 and 4, show larger discrepancies and are not suitable for the correct description of global quantities, such as the system power. It is thus interesting to compare the quality of the various point kinetic results in describing the response in the detector positions, close to the source as well as further away $(0.9 \mathrm{~L}$ and $5.3 \mathrm{~L})$. The results concerning the prediction of local fluxes are presented in Figures 5 and 6. To simplify the analysis of the results, the shape is adopted as the source-driven neutron distribution within the system, and the attention is focused on the type of point kinetic model that can be constructed by adopting different choices for the weight. In Figure 7, the graphs of the functions adopted as weights for this analysis are presented.

One can immediately notice how the quality of the point kinetic results is deteriorating when the source is far from the detector. On passing, it is worth also noticing that $l p k$ model results are characterized by a better performance, especially in the preservation of the areas, thus $l p k$ looks more suitable if the area-ratio method [4] is to be used. In Table 1, the accuracy of the forecast of the areas below the pulse for different models with respect to the exact result is presented, showing how better results can be obtained by the lpk model. On the other hand, if the detector is very close to the source (see the left graphs of Figures 5 and 6), neutrons can reach the detector at the very early stage of the transient and, therefore, the contribution of higher-order harmonics can be relevant.

\section{Time Modelization of the Effective Source}

In the derivation of the lumped-parameter (point) model through a projection procedure, only an integration over phase space is performed, thus leaving the time behaviour of the source unchanged. Therefore, no propagation effects associated to the finite velocity of neutrons can be accounted for. Of course the signal from localized detectors is affected 


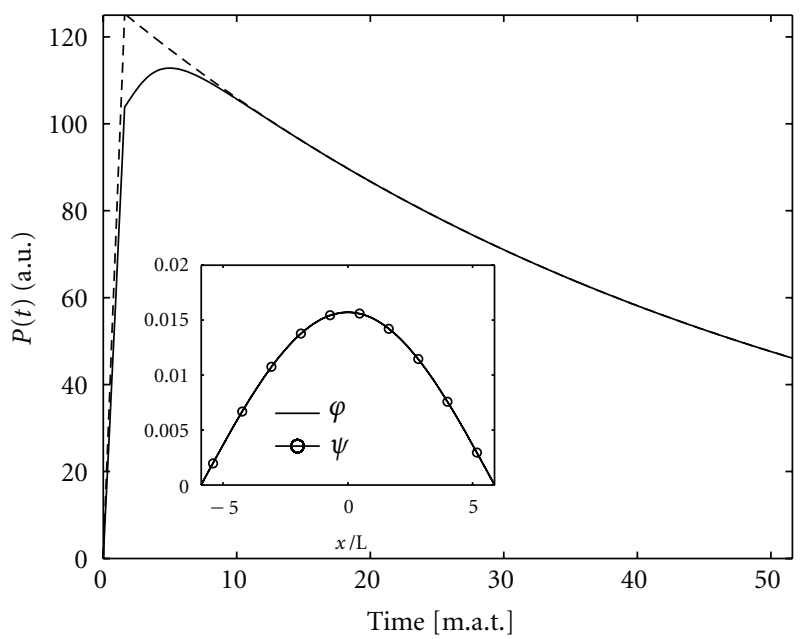

(a) $c p k$ and critical shape

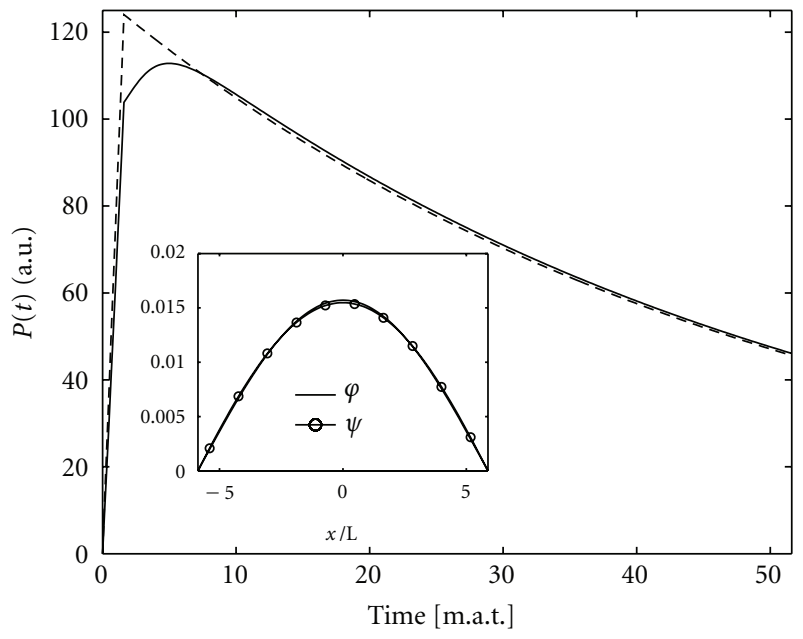

(c) $g p k$ and critical shape

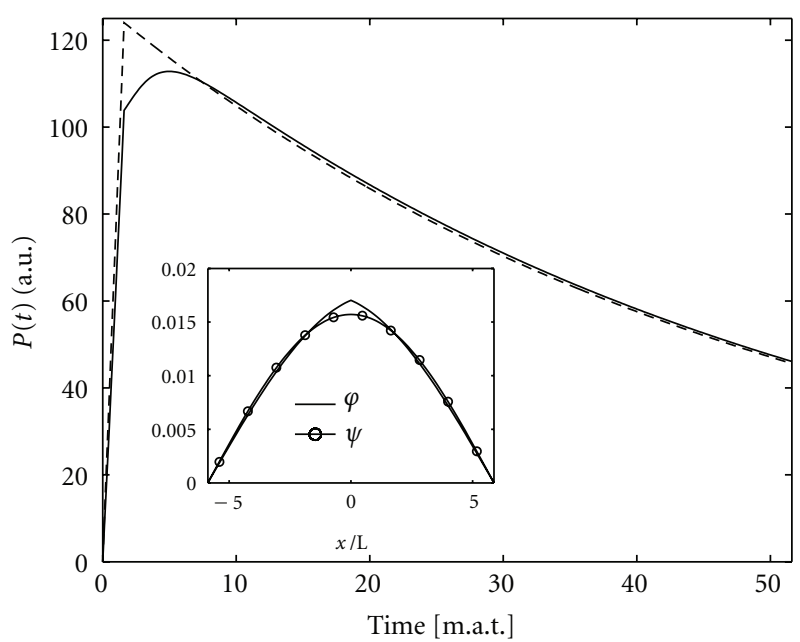

(b) $c p k$ and source-driven shape

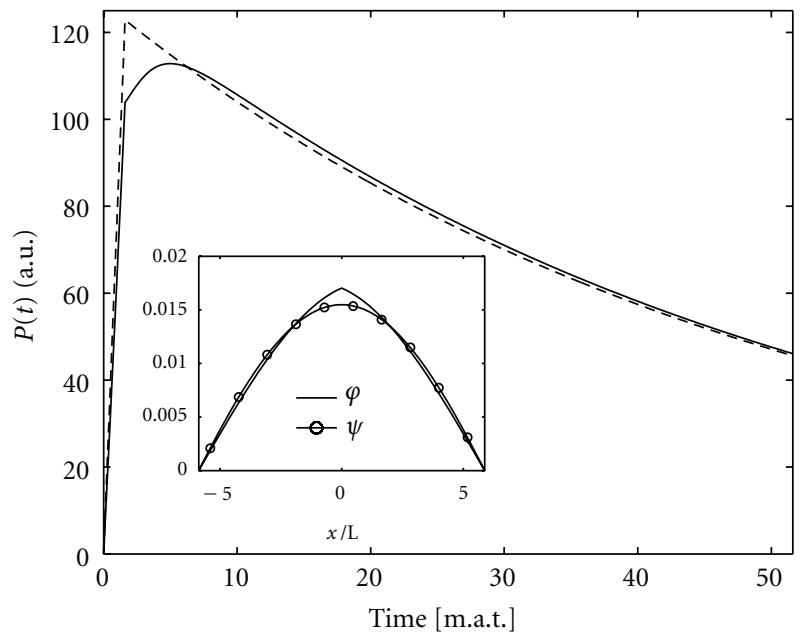

(d) $g p k$ and source-driven shape

FIGURE 3: Power evolution following a source pulse in a subcritical system $\left(k_{\text {eff }}=0.98\right)$, simulated with different approaches to point kinetics: $c p k$ and $g p k$. Solid line: reference; dashed line: point kinetics. The inset graphs represent the shape $\varphi$ and the weight $\psi$ adopted.

TABLE 1: Percentage relative error on the integral of the flux in the detector positions, as obtained by the different point kinetic models with respect to the reference solution. In bold the "best" results are evidenced.

\begin{tabular}{lccccc}
\hline \multirow{2}{*}{ PK model } & & \multicolumn{2}{c}{$k_{\text {eff }}=0.95$} & \multicolumn{2}{c}{$k_{\text {eff }}=0.98$} \\
& & $x_{0}=0.9 \mathrm{~L}$ & $x_{0}=5.3 \mathrm{~L}$ & $x_{0}=0.9 \mathrm{~L}$ & $x_{0}=5.3 \mathrm{~L}$ \\
\hline \multirow{2}{*}{ Source-driven } & $l p k$ & 0.66 & -2.29 & $\mathbf{0 . 1 2}$ & $\mathbf{0 . 2 9}$ \\
shape & $g p k$ & -0.92 & $\mathbf{1 . 2 2}$ & -1.83 & 3.90 \\
& $c p k$ & $-\mathbf{0 . 4 4}$ & 1.71 & -1.27 & 4.49 \\
\hline \multirow{5}{*}{ Critical shape } & $l p k$ & $\mathbf{0 . 1 7}$ & $\mathbf{1 . 7 1}$ & $-\mathbf{1 . 0 1}$ & $\mathbf{4 . 4 9}$ \\
& $g p k$ & -7.83 & 18.80 & -4.48 & 11.25 \\
& $c p k$ & -5.56 & 21.72 & -3.50 & 12.40 \\
\hline
\end{tabular}

by the distance of the detector with respect to the source and the effect is of particular relevance when a highly localized source is considered. In these cases, one may study the possibility to introduce in the effective source of the point model a suitable time delay.

Different options are considered to modify the time behaviour of the source in order to account for the time delay from the source to the detector. The delay may be assumed to be equal to the traveling time between the source and the detector or to an average displacement time on a suitable distribution, to be specified. A possible alternative is also the generation of the time behaviour by a convolution of the external source with the system response Green function. This idea is analogous to the practice of using the firstcollision distribution for studying the response to a highly localized pulsed source in transport problems.

The procedure to modify the source behaviour to account for the finite time propagation of the neutron signal seems somewhat arbitrary. Its motivation is just to try to capture the physical phenomenon connected to the propagation of the neutrons from the source to the detector, and, as such, it is problem dependent. The introduction into the point 


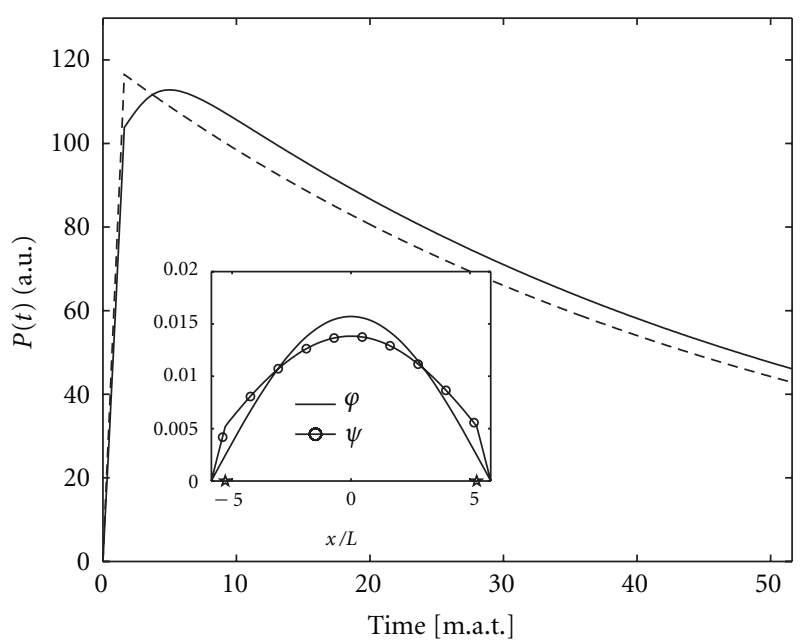

(a) $l p k$ and critical shape ( $\star$ : detector location)

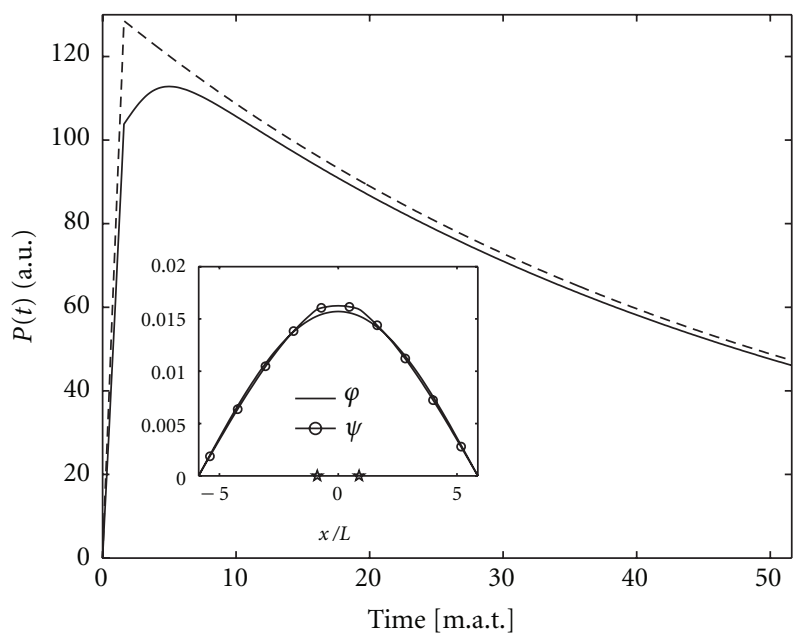

(c) lpk and critical shape ( $\star$ : detector location)

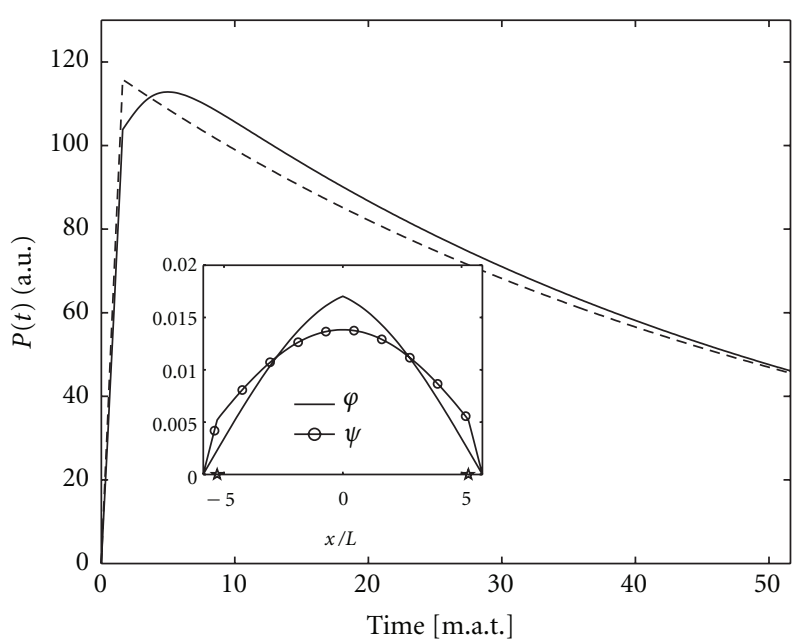

(b) $l p k$ and source-driven shape ( $\star$ : detector location)

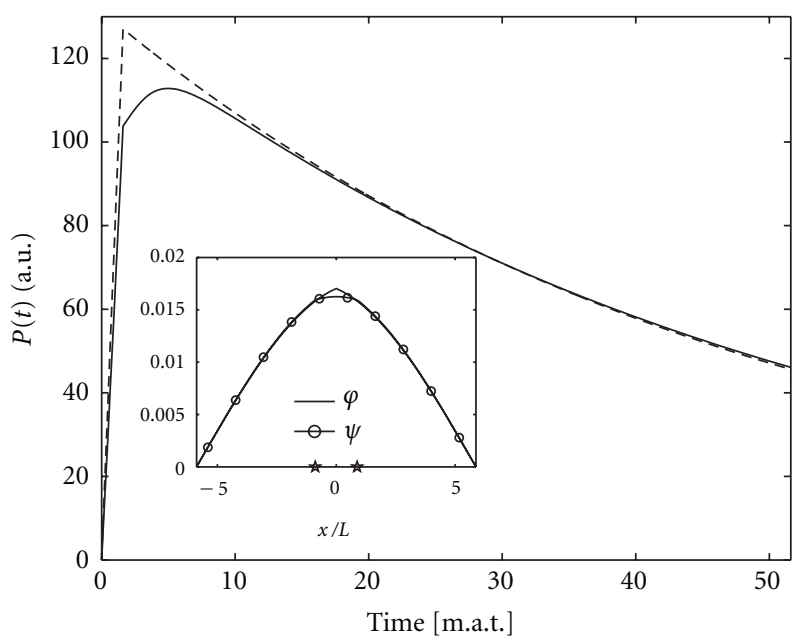

(d) lpk and source-driven shape ( $\star$ : detector location)

Figure 4: Power evolution following a source pulse in a subcritical system $\left(k_{\text {eff }}=0.98\right)$, simulated with a $l p k$ approach, considering two different detector locations: first row, detector far from the source $\left(x_{0}=5.3 \mathrm{~L}\right)$; second row: detector close to the source $\left(x_{0}=0.9 \mathrm{~L}\right)$. Solid line: reference; dashed line: point kinetics. The inset graphs represent the shape $\varphi$ and the weight $\psi$ adopted.

model is mathematically unjustified and an attempt to make it more consistent is certainly required, which lies in future developments and extensions of the present work.

The possible options are summarized in the following. For the standard point model, the effective source is assumed to be defined through the projection operation of the reference balance model. Therefore, its time dependency is given by

$$
S(t)=\frac{\langle\psi \mid S\rangle}{\langle\psi \mid \varphi\rangle}
$$

The time behaviour of the effective source may be modified when describing a detector at distance $x_{0}$ from the source by delaying the signal of the transit time for the neutrons between the source and the detector, that is, $\tau=x_{0} / \mathrm{v}$ (modification 1). Of course, this amounts to assuming an irrelevant contribution from the emitted neutrons from collisions taking place along the path from the source to the detector. In this case, the modified effective source $\tilde{S}$ is defined by the following formula:

$$
\tilde{S}(t)=S(t) u(t-\tau),
$$

where $u$ is the unitary Heaviside step function.

A further option involves the introduction of a delay time $\tau$ given by the average displacement time according to a given distribution, as

$$
\tau=\int d t^{\prime} f\left(x_{0}, t^{\prime}\right) t^{\prime}
$$

(modification 2). Alternatively, the source can be constructed as a convolution of the physical source on the proper Green 


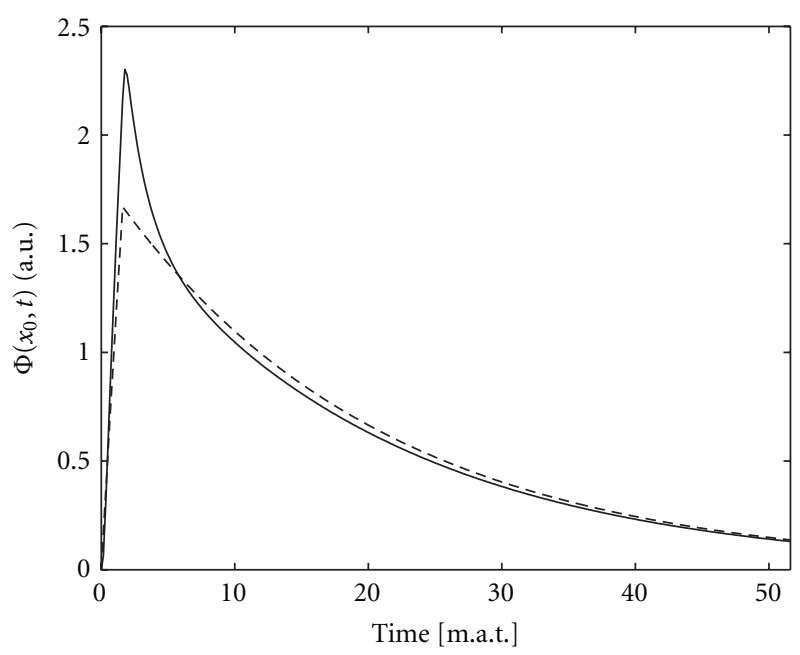

(a) $c p k$, detector at $0.9 \mathrm{~L}$

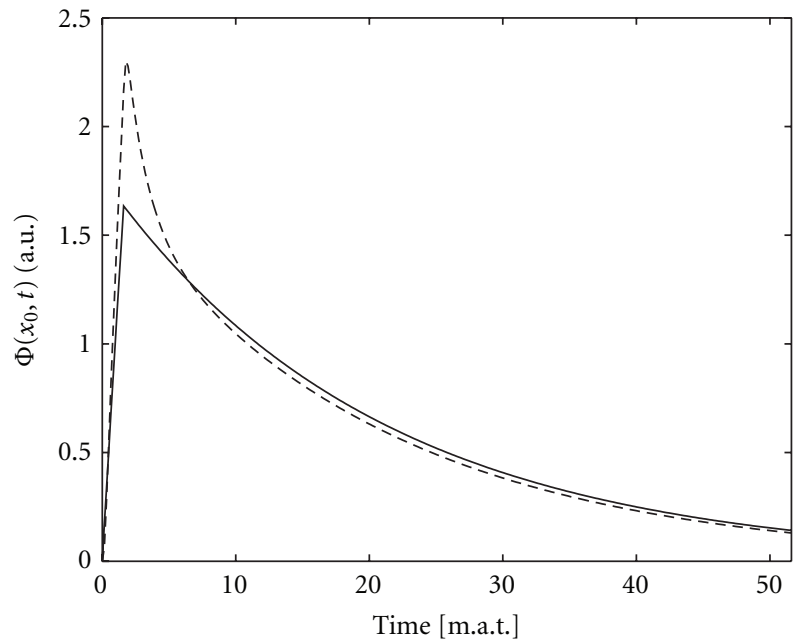

(c) $g p k$, detector at $0.9 \mathrm{~L}$

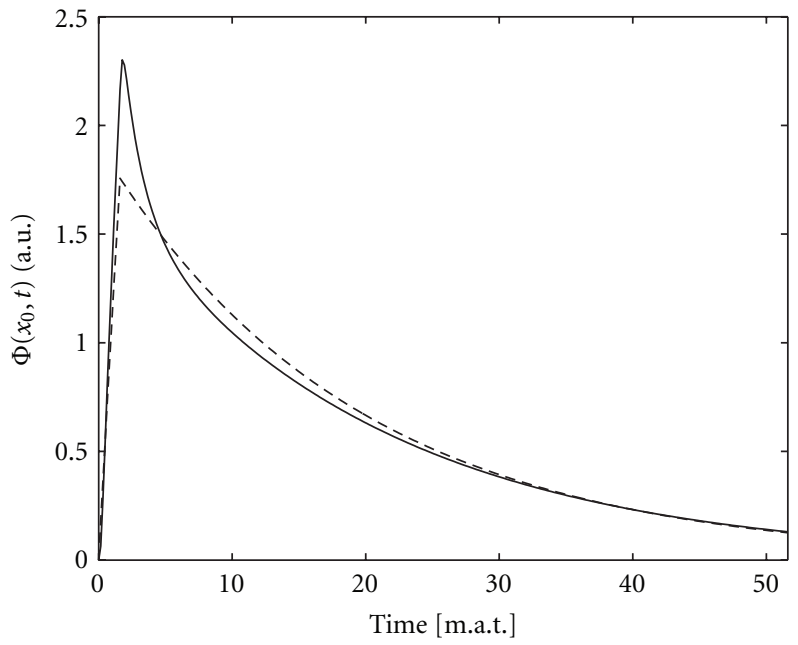

(e) $l p k$, detector at $0.9 \mathrm{~L}$

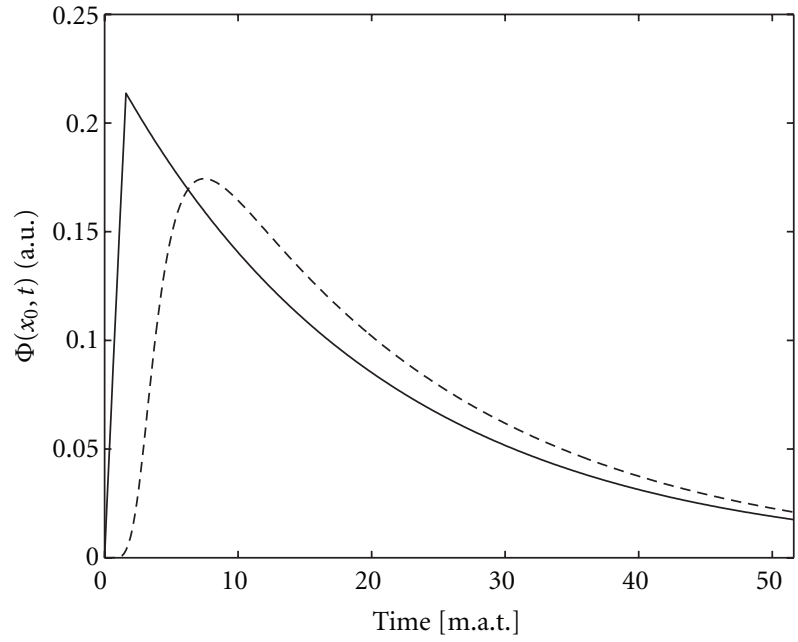

(b) $c p k$, detector at $5.3 \mathrm{~L}$

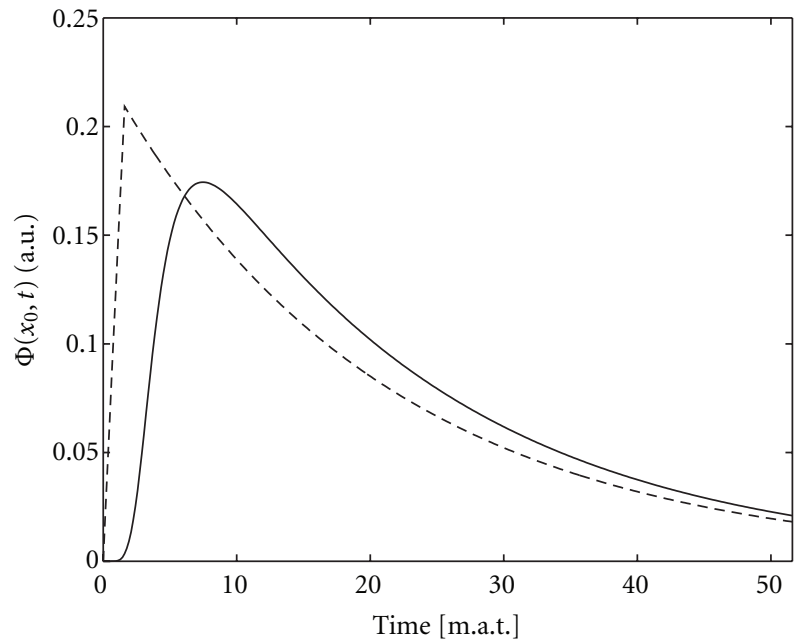

(d) $g p k$, detector at $5.3 \mathrm{~L}$

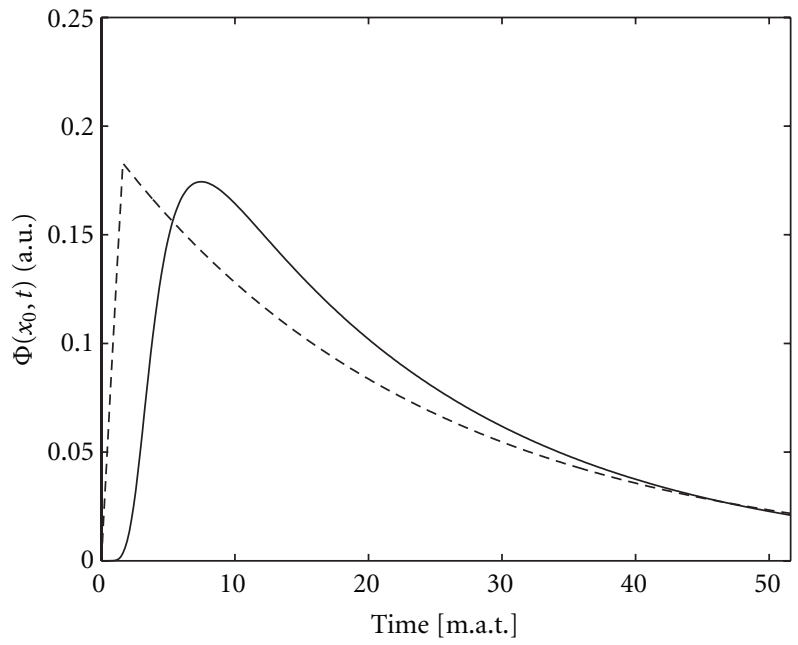

(f) $l p k$, detector at $5.3 \mathrm{~L}$

FIGURE 5: Evolution of the local flux following a source pulse in a subcritical system $\left(k_{\text {eff }}=0.95\right)$ for two detectors, at $x_{0}=0.9 \mathrm{~L}(\mathrm{a}, \mathrm{c}$, and e) and at $x_{0}=5.3 \mathrm{~L}(\mathrm{~b}, \mathrm{~d}$, and $\mathrm{f}$ ), adopting different formulations of the point kinetic model. Solid line: reference; dashed line: point kinetics. 


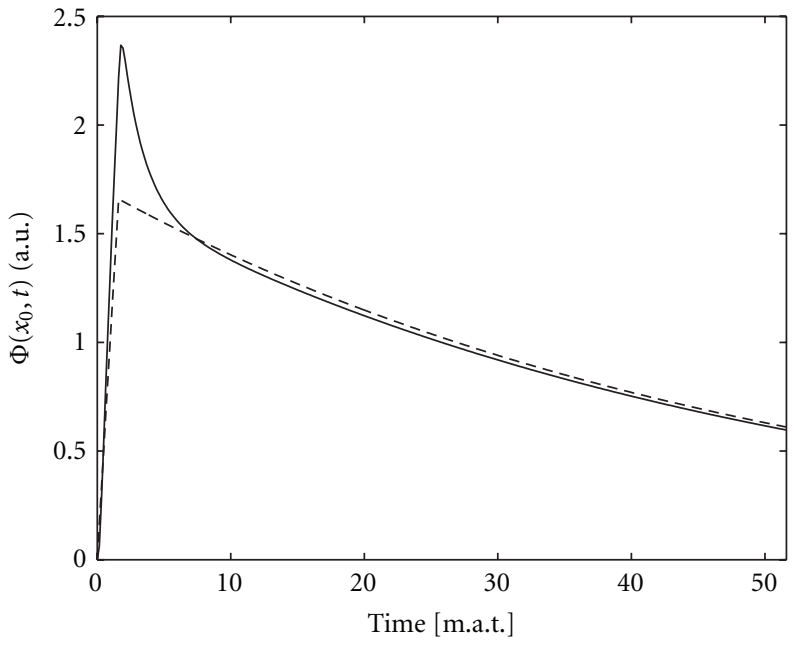

(a) $c p k$, detector at $0.9 \mathrm{~L}$

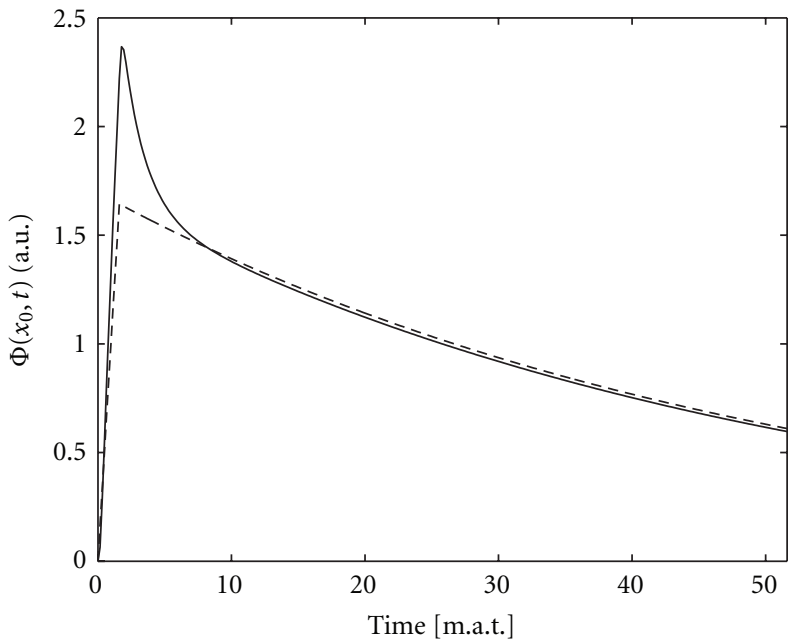

(c) $g p k$, detector at $0.9 \mathrm{~L}$

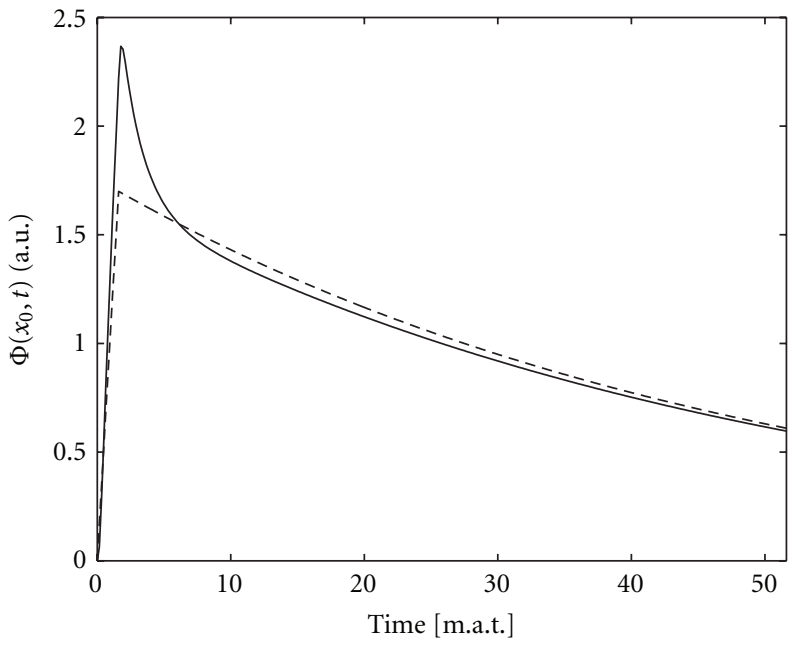

(e) $l p k$, detector at $0.9 \mathrm{~L}$

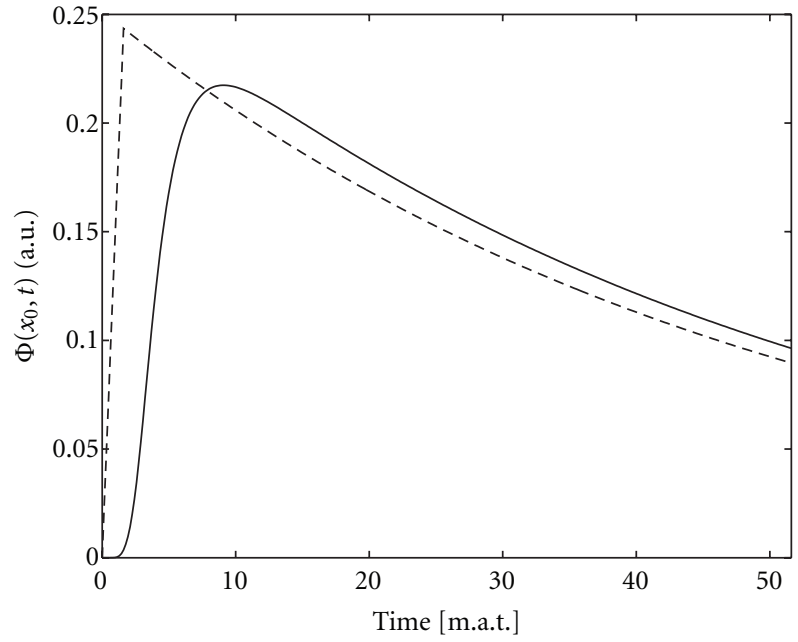

(b) $c p k$, detector at $5.3 \mathrm{~L}$

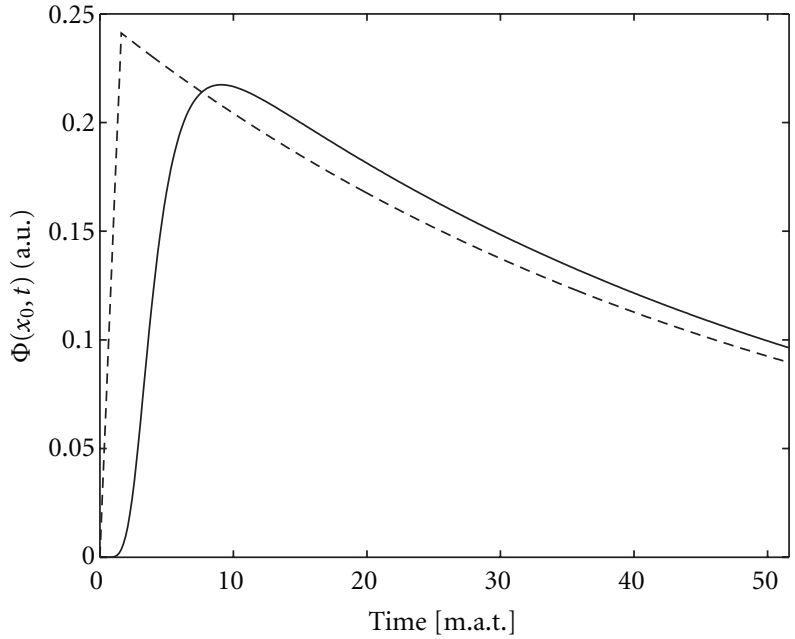

(d) $g p k$, detector at $5.3 \mathrm{~L}$

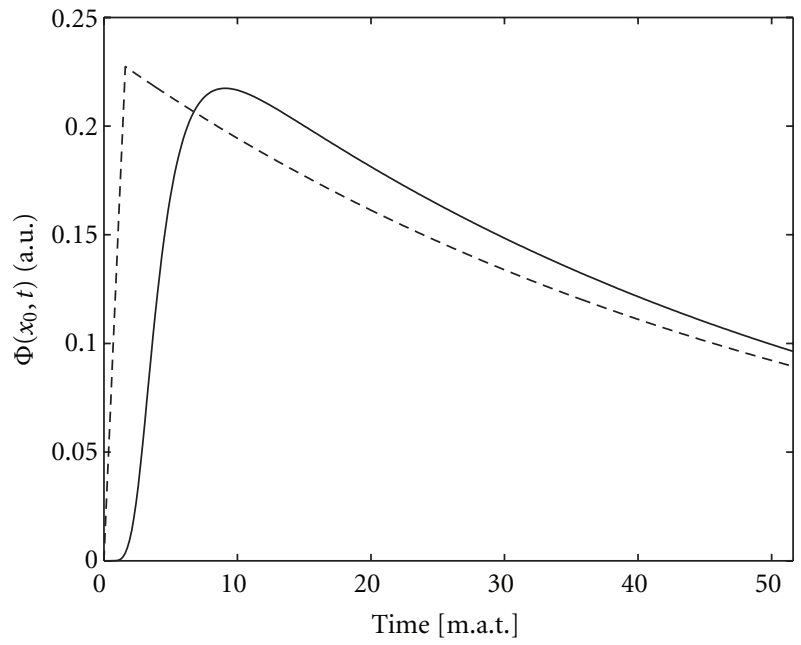

(f) $l p k$, detector at $5.3 \mathrm{~L}$

FIGURE 6: Evolution of the local flux following a source pulse in a subcritical system $\left(k_{\text {eff }}=0.98\right)$ for two detectors, at $x_{0}=0.9 \mathrm{~L}(\mathrm{a}, \mathrm{c}$, and e) and at $x_{0}=5.3 \mathrm{~L}(\mathrm{~b}, \mathrm{~d}$, and $\mathrm{f}$ ), adopting different formulations of the point kinetic model. Solid line: reference; dashed line: point kinetics. 


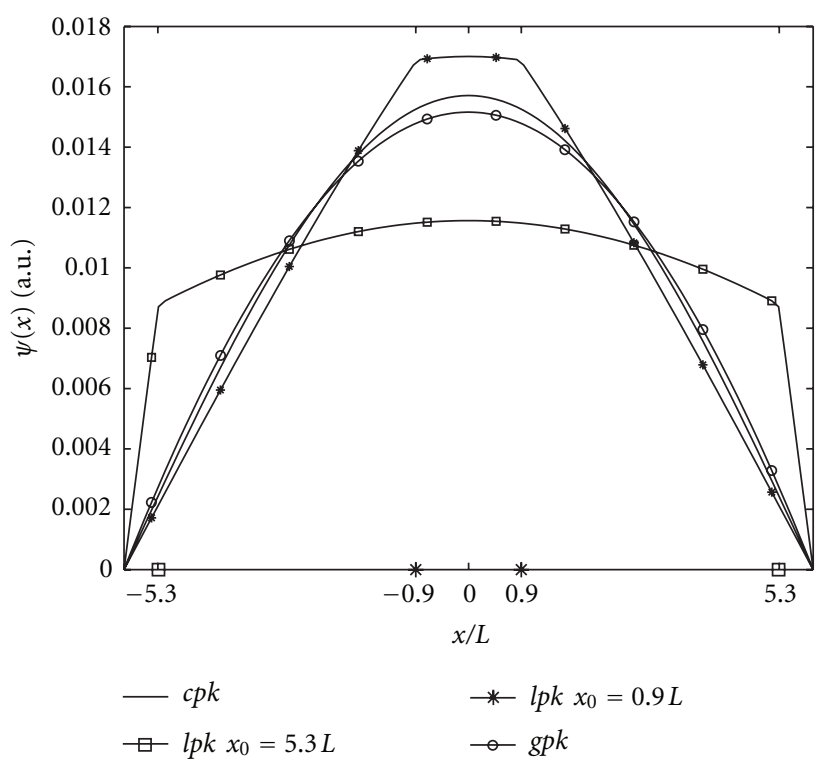

(a) $k_{\text {eff }}=0.95$

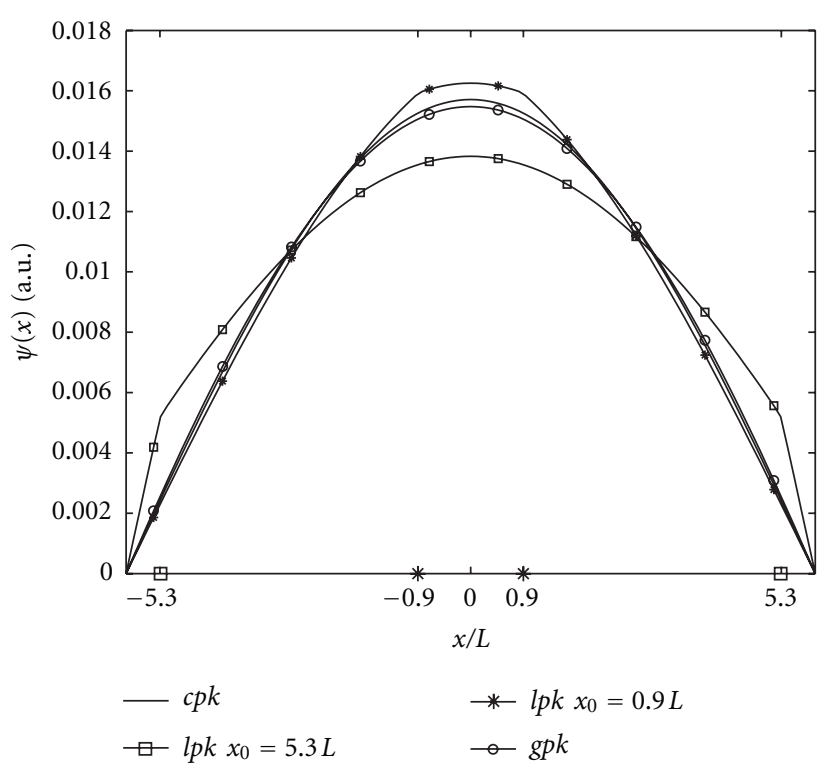

(b) $k_{\text {eff }}=0.98$

FIGURE 7: Weights adopted for the generation of the point kinetic models for the prediction of the local fluxes.

TABLe 2: Performance of $g p k$ and $l p k$ for the prediction of the system power during a transient induced by localized perturbations. Values of $\Delta P$ are computed as relative percentage errors with respect to the corresponding reference values at two different time instants along the transient $\left(t_{1}=1 \mathrm{~ms}\right.$ and $\left.t_{2}=10 \mathrm{~ms}\right)$.

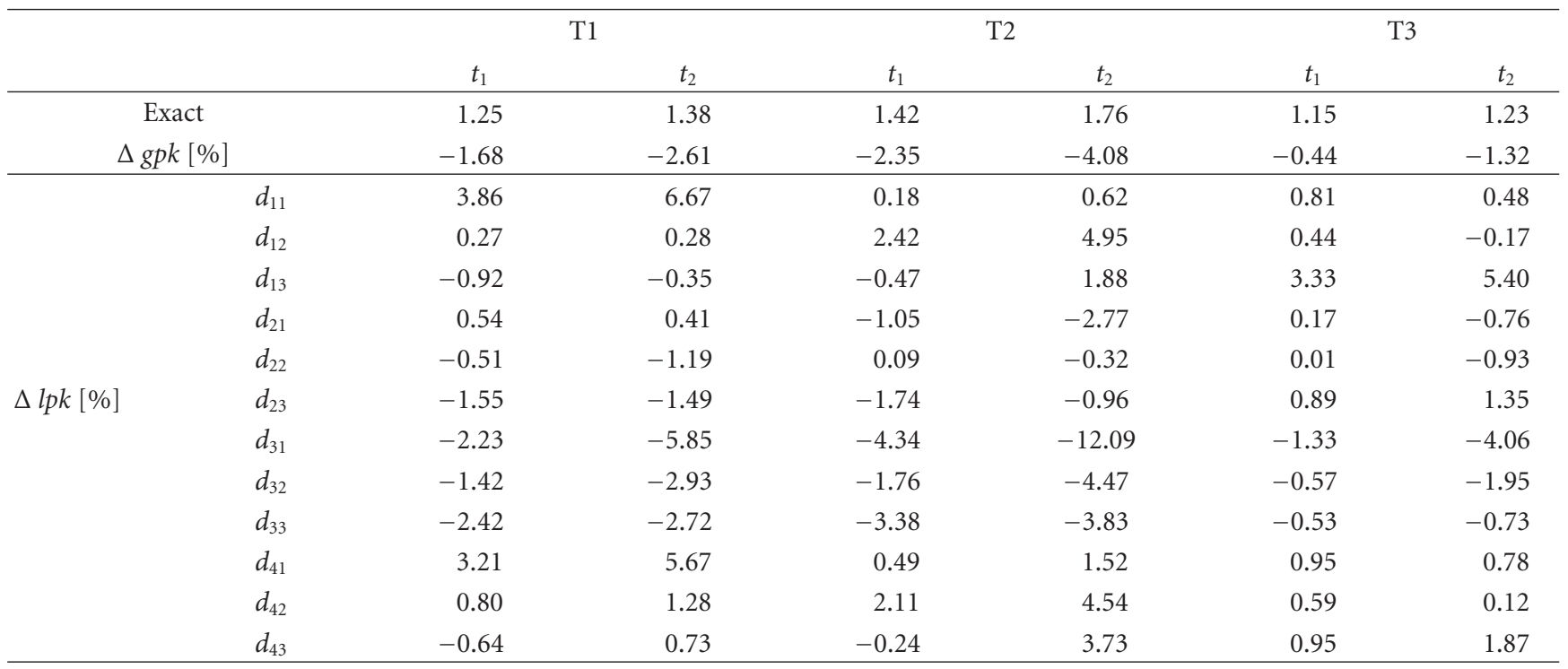

function of the problem (modification 3). This option amounts to the following choice:

$$
\tilde{S}(t)=\int d t^{\prime} S\left(t^{\prime}\right) G\left(x_{0}, t-t^{\prime}\right) .
$$

Definition (8) provides a better physical description of the migration process of the particles. In Figure 8, time behaviors of the source adopted for the calculations are compared, considering the position of the two detectors as in the previous evaluations. Both the system characteristics and the duration of the source pulse are the same as in all other calculations. The average displacement time is obtained using as a weighting distribution the Green function itself, after a proper renormalization in order to obtain a probability density function. The relevant modification in the time behavior of the source when a convolution with the Green function is adopted is evident. Figure 9 illustrates the reproduction of the response to a pulse with the above source assumptions for the two detectors (locations at $5.3 \mathrm{~L}$ and $0.9 \mathrm{~L}$ from the source). Good results can be obtained using the third modification for a signal far from the source and also a good simulation of the decaying portion of the 
TABle 3: Performance of $g p k$ and $l p k$ for the prediction of the flux in detectors located at $x_{\alpha}$ (see positions in Figure 10(b)) during a transient induced by localized perturbations. Values of $\Delta \varphi$ are computed as relative percentage errors with respect to the corresponding reference values at two different time instants along the transient $\left(t_{1}=1 \mathrm{~ms}\right.$ and $\left.t_{2}=10 \mathrm{~ms}\right)$.

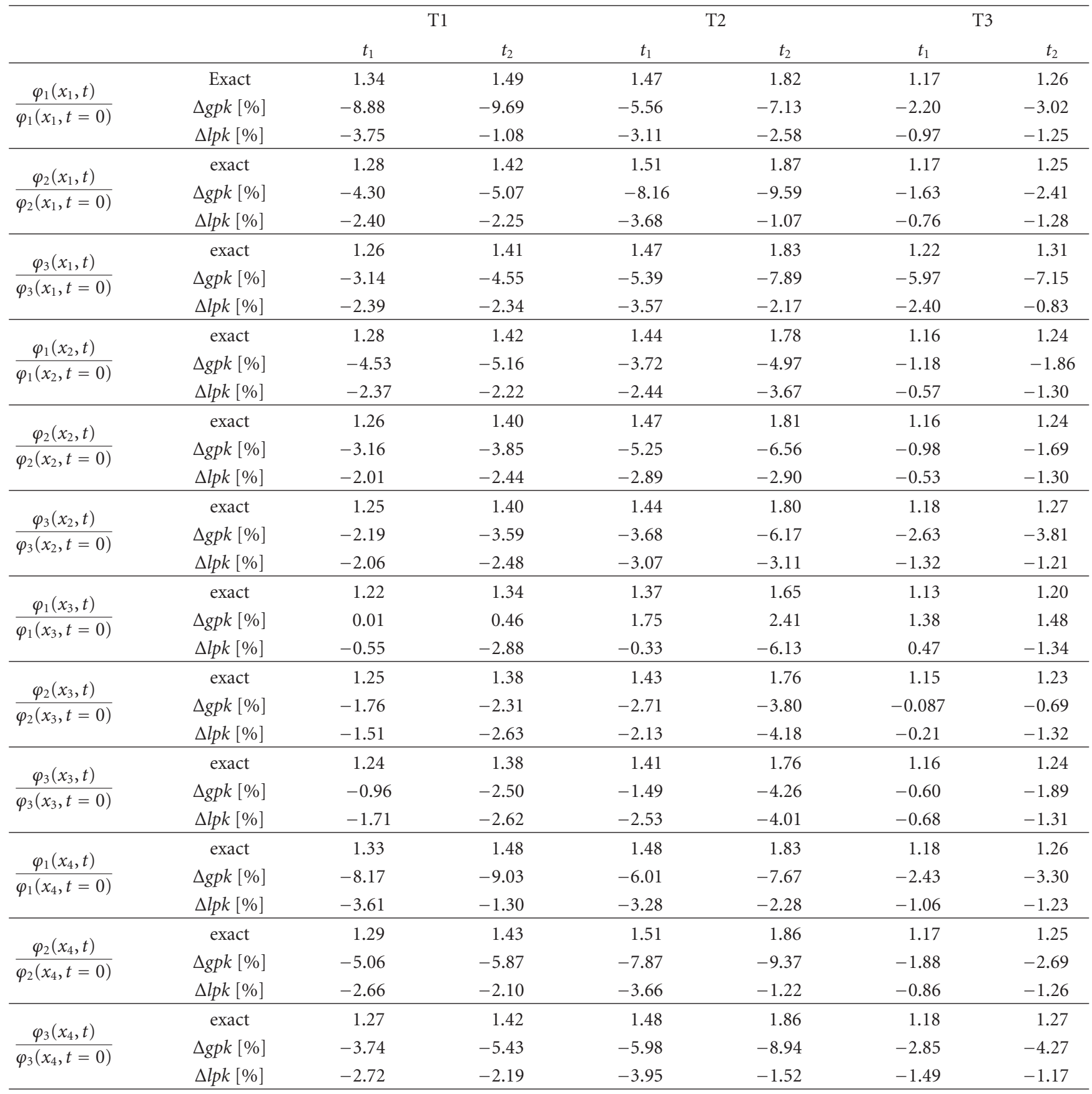

curve is reached, which is needed for the application of several interpretation procedures. On the other hand, a little improvement can be obtained when the detector is very close to the source. In this case, it is important also to recall that the presence of high transport effects dominated by the uncollided fraction of the source neutrons makes the lumped-parameter and the whole source-delay formalism inapplicable.

\section{Use of Local Point Kinetics for Transient Simulation}

The potentialities of improvement adopting a point kinetic model with a problem-tailored weighting are now tested for the simulation of transients in a subcritical system. Results are compared with those produced using a standard global weighting, that is, assuming as adjoint source the fission 
TABle 4: Percentage relative error on the flux at position $x_{1}$ and time $t=10 \mathrm{~ms}$ during transient $\mathrm{T} 1$ with different localizations of the adjoint source for the definition of the lpk model adopted. In bold, the result obtained when the adjoint source is located where the detector is (spatially and energetically).

\begin{tabular}{cccc}
\hline$S^{\dagger}$ & $\Delta \phi_{1}$ & $\Delta \phi_{2}$ & $\Delta \phi_{3}$ \\
\hline$d_{11}$ & $-\mathbf{1 . 1 0}$ & 4.00 & 4.54 \\
$d_{12}$ & -7.00 & -2.25 & -1.72 \\
$d_{13}$ & -7.59 & -2.87 & -2.34 \\
\hline$d_{21}$ & -6.89 & -2.12 & -1.59 \\
$d_{22}$ & -8.37 & -3.68 & -3.16 \\
$d_{23}$ & -8.65 & -3.98 & -3.46 \\
\hline
\end{tabular}

productivity $v \Sigma_{f}(g p k)$. Spectral effects can also be important; hence results of a three-group calculation are now presented. The evolution is induced in a fast reflected one-dimensional slab structure (see Figure 10), with material characteristics as for the system studied in [7], by a local perturbation of the absorption cross section in each one of the corresponding energy group inside the core to investigate also spectral effects. The location of the perturbed region is specified in Figure 10(a).

The features of the different point kinetic models can be clearly seen by observing Tables 2 and 3 , where the differences between power level and local fluxes obtained with the use of $g p k$ and $l p k$ at two different time instants $\left(t_{1}=1 \mathrm{~ms}\right.$ and $t_{2}=10 \mathrm{~ms}$ ) during the transients are reported. All results are obtained by comparison with a reference produced by a full space-time solution. The system starts from an initial multiplication constant $k_{\text {eff }}=0.975$, and three transients are considered $(\mathrm{T} j, j=1,2,3)$, where the index $j$ indicates in which energy group the cross section perturbation is introduced. The reactivity insertions associated to each transient are $811 \mathrm{pcm}, 1263 \mathrm{pcm}$, and $552 \mathrm{pcm}$, respectively. The detector $d_{\alpha g}$ is localized at position $x_{\alpha}$ and detects neutrons belonging to energy group $g$. The detectors are localized in the center of the perturbed region $\left(x_{1}=60.9 \mathrm{~cm}\right)$, outside the perturbation within the fissile region $\left(x_{2}=71.5 \mathrm{~cm}\right)$, in the lead zone close to the source $\left(x_{3}=82.1 \mathrm{~cm}\right)$, and in the reflector $\left(x_{4}=41.9 \mathrm{~cm}\right)$, see Figure 10(b).

Point kinetic models based on a global weighting always underestimate the power signal, as shown in Table 2. The performances of point models with a detector-based adjoint source, when adopted for the power prediction, provide results with different ranges of error, thus not evidencing a clear pattern. It is thus of interest to compare the performance of such models in the reproduction of the local flux signals, summarized in Table 3. It can be observed how $l p k$ is usually providing a smaller error with respect to $g p k$ when the adjoint source is located in the position where the flux is observed, since it can capture the physical information most effectively.

It is also of interest to see how the performance can be affected by a less proper choice of the detector. In Table 4 , the attention is focused on the flux in the detector 1 (within the

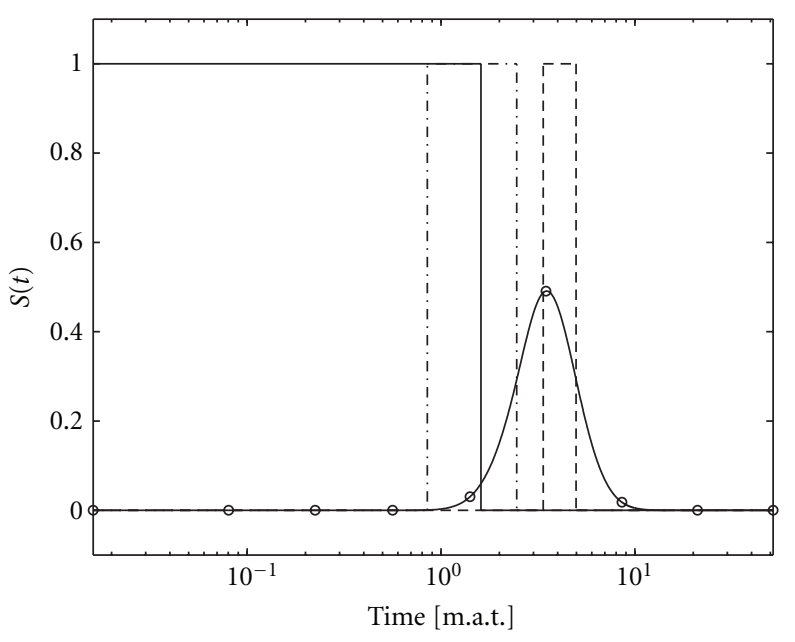

$\begin{aligned}-\cdot-\tau & =5.3 \mu \mathrm{s} \\ --\tau & =21 \mu \mathrm{s}\end{aligned}$

(a) $x_{0}=5.3 \mathrm{~L}$

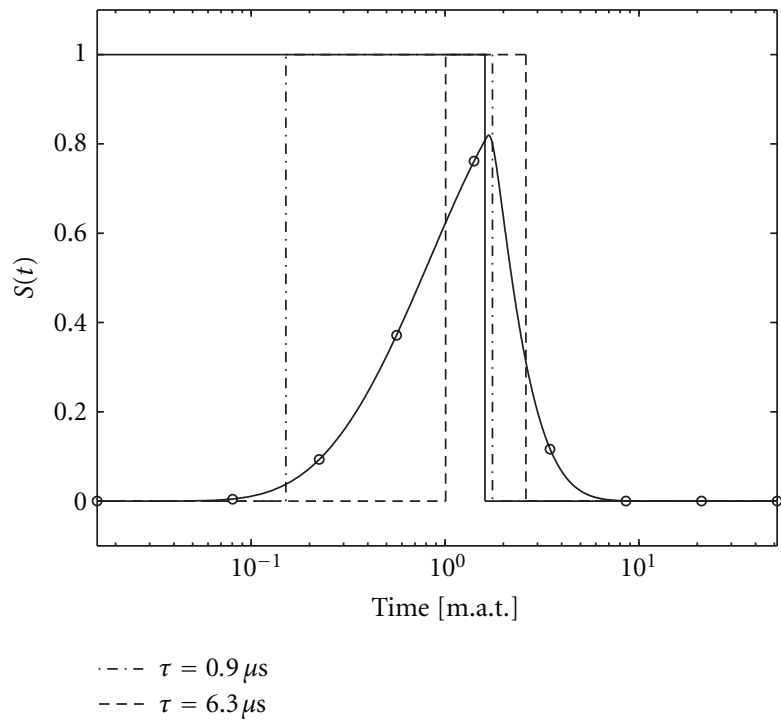

(b) $x_{0}=0.9 \mathrm{~L}$

FIGURE 8: Time behavior of the sources according to the modifications proposed. Solid: original case; dash-dotted: modification 1; dashed: modification 2; circles: modification 3.

perturbed region) and is simulated with different $l p k$ models, placing the adjoint source in the perturbed region and in the correct energy range (first group), and then moving it to different spatial and spectral locations. The degradation of the quality of the results when the adjoint source moves further from the position of observation is quite evident.

At last, an evaluation of the reactivity term from $l p k$ and $g p k$, compared to the subcriticality level in the system, is performed. The same configuration in three-group diffusion as in the previous calculations is considered, imposing different levels of subcriticality in order to test the performances for configurations close and far from criticality. In Table 5, the 
TABLE 5: Error in pcm on the subcriticality level as obtained by the different point kinetic models adopted in the one-dimensional domain sketched in Figure 10. The direct shape is assumed to be the subcritical source-driven distribution.

\begin{tabular}{|c|c|c|c|c|c|c|c|}
\hline \multirow{5}{*}{$\begin{array}{l}\rho_{0}=-7526.9 \\
\rho_{0}\end{array}$} & \multirow{5}{*}{$\begin{array}{l}\Delta g p k[\mathrm{pcm}] \\
12.9\end{array}$} & \multicolumn{6}{|c|}{$\Delta l p k[\mathrm{pcm}]$} \\
\hline & & $d_{11}$ & $d_{12}$ & $d_{13}$ & $d_{21}$ & $d_{22}$ & $d_{23}$ \\
\hline & & 337.0 & 213.1 & 111.1 & -262.4 & -47.7 & -55.2 \\
\hline & & $d_{31}$ & $d_{32}$ & $d_{33}$ & $d_{41}$ & $d_{42}$ & $d_{43}$ \\
\hline & & -3742.5 & -473.7 & -202.7 & 462.9 & 304.7 & 244.3 \\
\hline \multirow{4}{*}{$\rho_{0}=-2564.1$} & \multirow{4}{*}{1.6} & $d_{11}$ & $d_{12}$ & $d_{13}$ & $d_{21}$ & $d_{22}$ & $d_{23}$ \\
\hline & & 42.4 & 26.6 & 13.9 & -32.8 & -6.3 & -7.0 \\
\hline & & $d_{31}$ & $d_{32}$ & $d_{33}$ & $d_{41}$ & $d_{42}$ & $d_{43}$ \\
\hline & & -458.0 & -58.8 & -25.2 & 58.4 & 38.3 & 30.7 \\
\hline \multirow{4}{*}{$\rho_{0}=-1010.1$} & \multirow{4}{*}{0.3} & $d_{11}$ & $d_{12}$ & $d_{13}$ & $d_{21}$ & $d_{22}$ & $d_{23}$ \\
\hline & & 6.8 & 4.2 & 2.2 & -5.2 & -1.0 & -1.1 \\
\hline & & $d_{31}$ & $d_{32}$ & $d_{33}$ & $d_{41}$ & $d_{42}$ & $d_{43}$ \\
\hline & & -72.3 & -9.3 & -4.0 & 9.3 & 6.1 & 4.9 \\
\hline
\end{tabular}

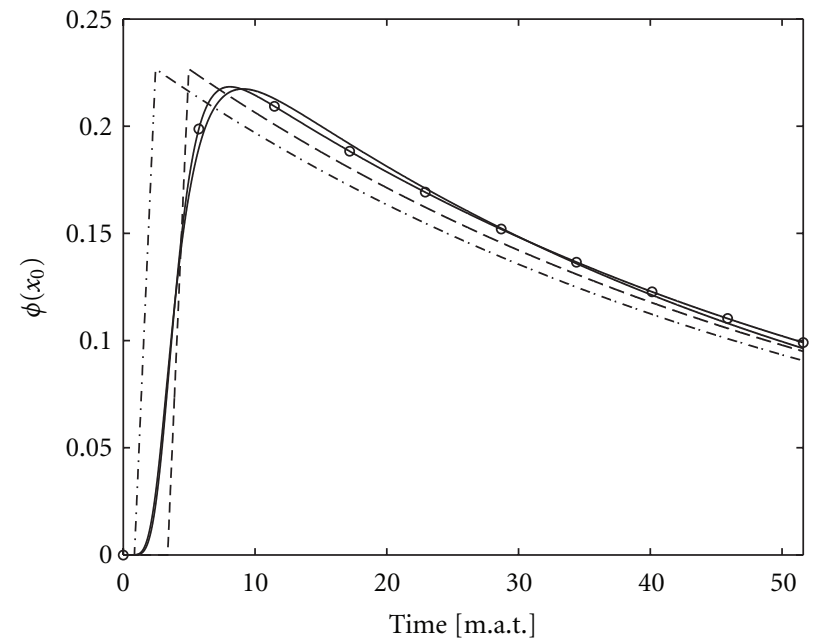

(a) $x_{0}=5.3 \mathrm{~L}$

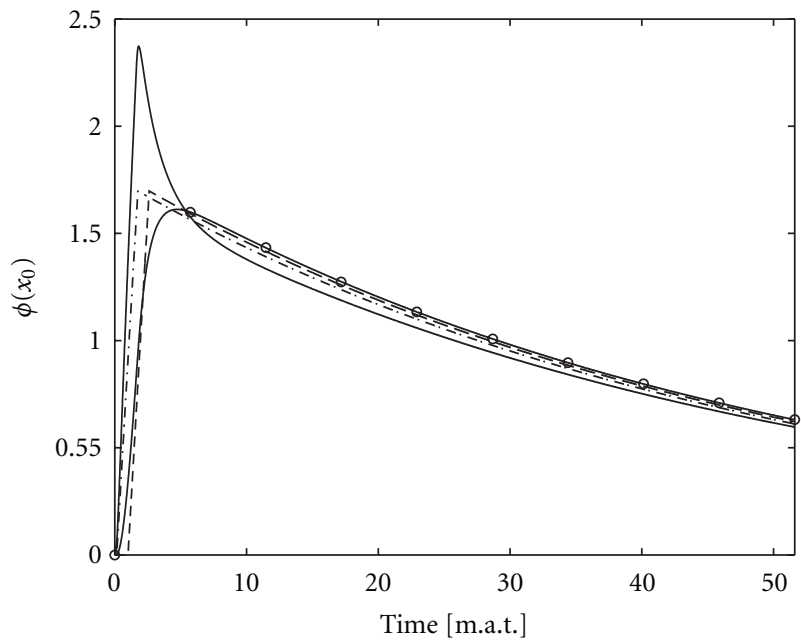

(b) $x_{0}=0.9 \mathrm{~L}$

FIGURE 9: Local flux response to a source pulse obtained with point kinetics in association with a modified treatment of the source time behavior. Solid: reference; dash-dotted: modification 1; dashed: modification 2; circles: modification 3.

errors on $\rho_{0}$ in pcm $\left(10^{-5}\right)$ are reported. As expected, systems closer to criticality are associated to smaller discrepancies, and the adoption of a global weighting for the prediction of the subcriticality level appears to be more effective. The performances of local detectors again show different trends. However, it can be noticed that, when the energy localization of the detector corresponds to the thermal group, a better quality in the results can be achieved. This is due to the larger amount of information associated to neutrons that, after being emitted by fission or by the external source at high energy, are slowed down within the system and have a "better knowledge" of the subcritical configuration. A similar reasoning explains why the results produced by the detector closer to the source (detector 3) are of lower quality: this position detects primarily neutrons directly from the source, with little information of the subcritical assembly.

\section{Concluding Remarks}

Point models that satisfactorily represent the kinetic behavior of the local flux evolution in a subcritical system can be developed using different neutron shapes and, as weighting function for the projection, the adjoint solution of a sourcedriven problem where the local detector is used as a source. A better physical representation of the source can be attained by introducing suitable delay functions in the effective source term.

The limits and shortcomings of possible kinetic models are discussed, illustrating results for some simple but physically representative configurations, in the effort to evidence specific sources of errors and the space for improvements. It is shown that the choice of a local detector-tailored weighting is the most appropriate for experimental flux measurement interpretation. 


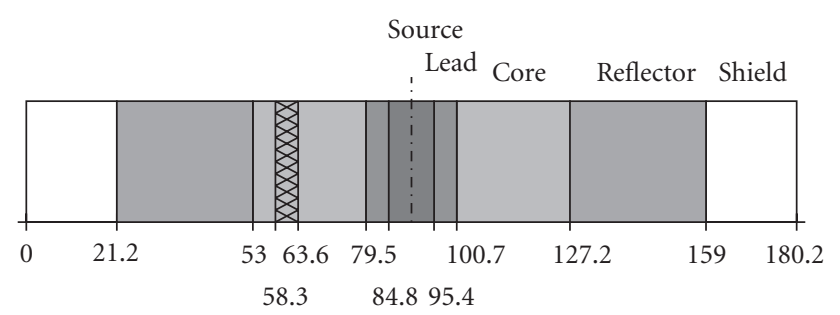

(a) domain subdivision

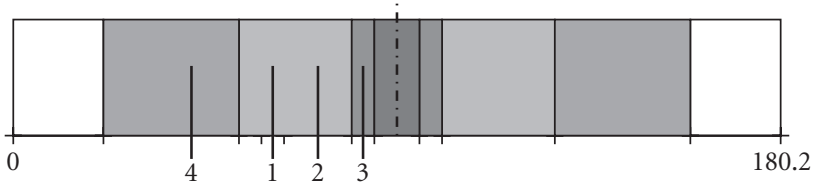

(b) detector localization

Figure 10: System domain adopted for the three-group calculations. The localization of the region where the cross section perturbations are introduced is highlighted (a), as well as the position of the four detectors (b).

Another important issue that is discussed concerns the proper treatment of the source term in the kinetic model, with special regards to its time behaviour. It is shown that the introduction of a suitable delay is necessary to adequately reproduce the system response.

In conclusion, the point model performs satisfactorily if local kinetic parameters are generated and if the source is properly delayed to account for propagation times inside the finite medium. In future works, the analysis of the point model presented in this work will be extended to more effective approaches, such as multipoint, with the objective to yield more accurate computational tools for kinetic experiment evaluations.

\section{References}

[1] R. Soule, W. Assal, P. Chaussonnet et al., "Neutronic studies in support of accelerator-driven systems: the MUSE experiments in the MASURCA facility," Nuclear Science and Engineering, vol. 148, no. 1, pp. 124-152, 2004.

[2] C. M. Persson, A. Fokau, I. Serafimovich et al., "Pulsed neutron source measurements in the subcritical ADS experiment YALINA-Booster," Annals of Nuclear Energy, vol. 35, no. 12, pp. 2357-2364, 2008.

[3] N. G. Sjöstrand, "Measurements on a subcritical reactor with a pulsed neutron source," in Proceedings of the International Conference on the Peaceful Uses of Atomic Energy, vol. 5, United Nations, New York, NY, USA, 1956.

[4] N. G. Sjöstrand, "Measurements on a subcritical reactor using a pulsed neutron source," Archiv fr Physik, vol. 11, pp. 233-246, 1956.

[5] I. Pázsit and C. Demazière, "Noise techniques in nuclear systems," in Handbook of Nuclear Engineering, D. G. Cacuci, Ed., vol. 2, pp. 1631-1734, Springer, New York, NY, USA, 2010.

[6] P. Ravetto, M. M. Rostagno, and P. Vinai, "Inverse problem for the determination of the reactivity in pulsed experiments for subcritical systems," Transactions of the American Nuclear Society, vol. 86, pp. 426-428, 2002.
[7] M. Carta, A. D’Angelo, V. Peluso et al., "Reactivity assessment and spatial time-effects from the MUSE kinetics experiments," in Proceedings of the International Conference: The Physics of Fuel Cycles and Advanced Nuclear Systems-Global Developments (PHYSOR '04), pp. 657-669, Chicago, Ill, USA, April 2004.

[8] M. Becker, "A generalized formulation of point nuclear reactor kinetics equations," Nuclear Science and Engineering, vol. 31, pp. 458-464, 1968.

[9] S. Dulla, P. Ravetto, M. M. Rostagno, G. Bianchini, M. Carta, and A. D'Angelo, "Some features of spatial neutron kinetics for multiplying systems," Nuclear Science and Engineering, vol. 149, no. 1, pp. 88-100, 2005.

[10] S. Dulla, F. Cadinu, and P. Ravetto, "Neutron importance in source-driven systems," in Proceedings of the International Topical Meeting on Mathematics and Computation (MeC '05), Avignon, France, 2005.

[11] S. Dulla, M. M. Rostagno, and P. Ravetto, "Models for the interpretation of local flux measurements in subcritical systems," Transactions of the American Nuclear Society, vol. 93, pp. 568-570, 2005.

[12] A. F. Henry, Nuclear-Reactor Analysis, MIT Press, Cambridge, Mass, USA, 1975.

[13] L. N. Ussachoff, "Equations for importance of neutrons, reactor kinetics and perturbation theory," in Proceedings of the International Conference on the Peaceful Uses of Atomic Energy, vol. 5, pp. 503-570, Geneva, Switzerland, 1955. 

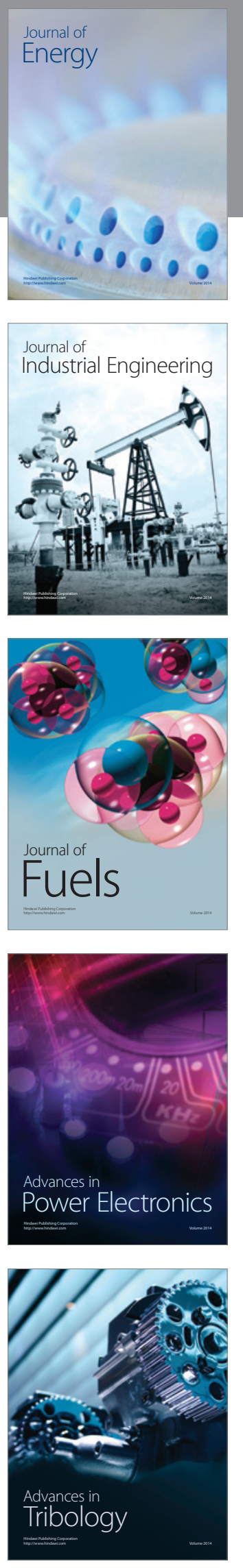
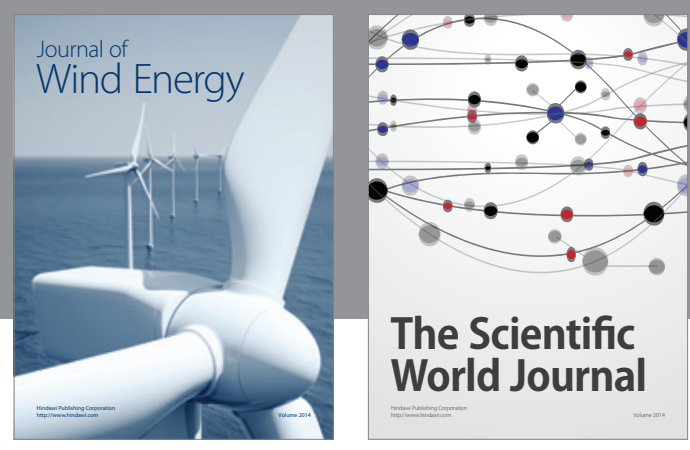

The Scientific World Journal

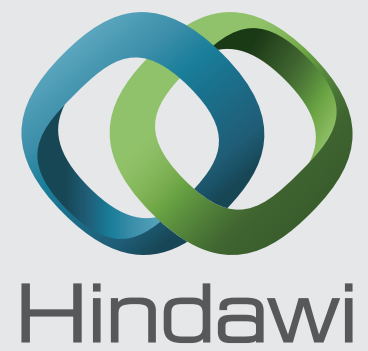

Submit your manuscripts at http://www.hindawi.com
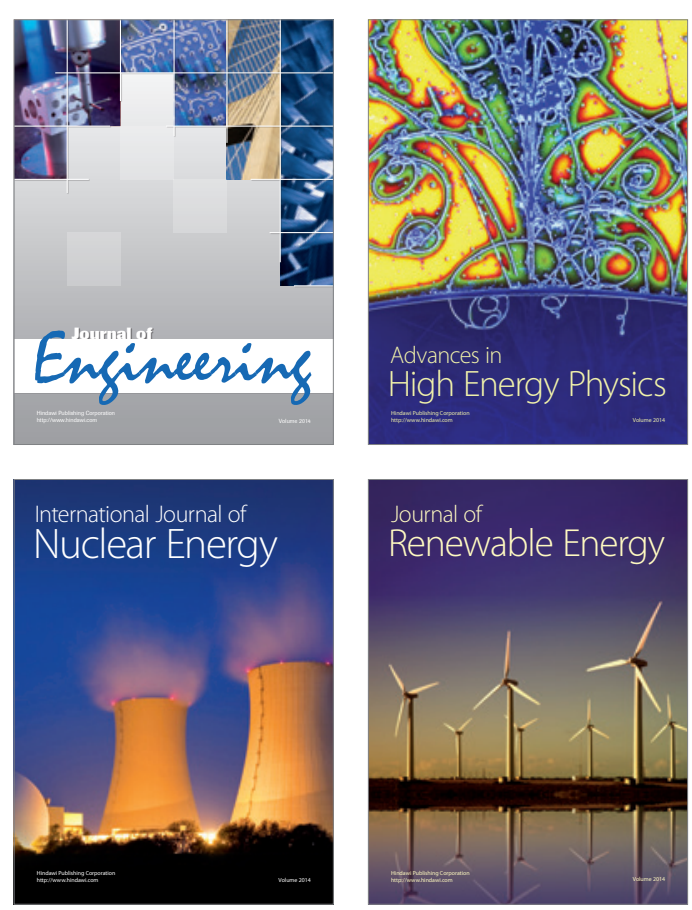

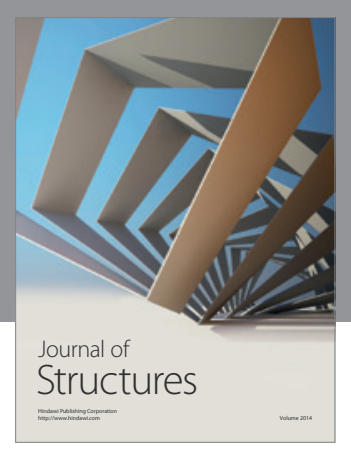

Rotating
Mechinery
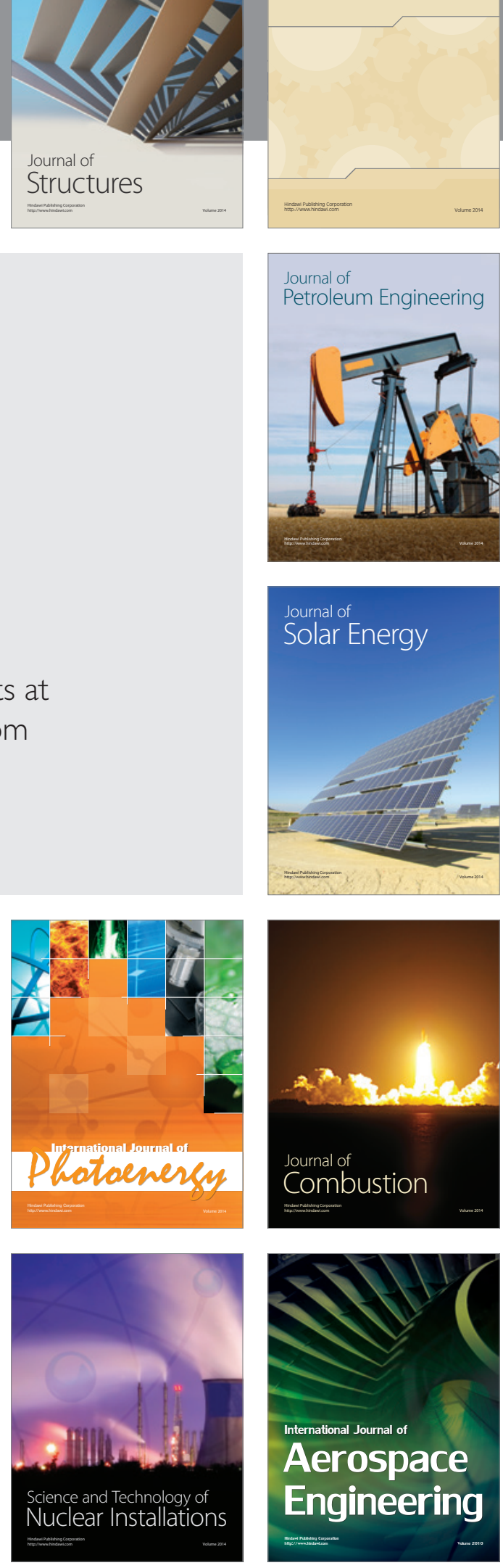